\title{
Vector Network Analyzer Techniques to Measure WR340 Waveguide Windows
}

T. L. Smith

ASD / RF Group

Advanced Photon Source

Argonne National Laboratory

June 26, 2002

\section{Table of Contents}

1) Introduction

2) Objective

3) Full Two-Port Calibration

4) TRL (Thru-Reflect-Line)

5) TRL / WR340 Waveguide Window Measurement

6) Conclusions

7) References

\section{Introduction}

In its fundamental form, network analysis involves the measurement of incident, reflected, and transmitted waves that travel along transmission lines. Measuring both magnitude and phase of components is important for several reasons. First, both measurements are required to fully characterize a linear network and ensure distortionfree transmission. To design effective matching networks, complex impedances must be measured. In the development of computer-aided-design (CAD) circuit simulation programs, magnitude and phase data are required for accurate models. In addition, timedomain characterization requires magnitude and phase information in order to perform an inverse Fourier transform [1].

To acquire accurate data using network analyzers special care must be taken when performing calibrations and measurements. Various calibrations and measurement techniques using a vector network analyzer (HP8510C) will be discussed. The design of a WR340 waveguide rf window will be used as an example for explaining some of these techniques. 


\section{Objective}

A major problem encountered when making network measurements is the need to separate the effects of the transmission medium from the device characteristics. While it is advantageous to be able to predict how a device will behave in the environment of its final application, it can be difficult to measure this way. In most microwave measurements, systematic errors are the most significant source of measurement uncertainty. Systematic errors are caused by imperfections in the test equipment and test setup. If these errors do not vary over time, they can be characterized through calibration and mathematically removed during the measurement process [2]. The process of removing systematic errors from the network analyzer S-parameter measurement is called "measurement calibration."

\section{Full Two-Port Calibration}

A measurement calibration is a process that mathematically derives the error model for the network analyzer. This error model is an array of vector coefficients used to establish a fixed reference plane of zero phase shift, zero magnitude, and known impedance. A full two-port error model is only one example of the measurement calibrations available with the HP8510. The measurement calibration process for the HP8510 must be one of seven types: Response, Response \& Isolation, S1 1 1-Port, S22 1-Port, One Path 2-Port, Full 2Port, and TRL 2-Port [3]. The type of measurement calibration selected by the user depends on the device to be measured and the extent of accuracy desired. A combination of calibrations can be used in the measurement of a particular device.

A "Full 2-Port" includes both forward and reverse error terms. For this calibration, the standards from a calibration kit would be applied to both ports during the calibration procedure. A calibration kit is a set of physical devices called standards. Each standard has a precisely known or predictable magnitude and phase response as a function of frequency. In order for the HP8510 to use the standards of a calibration kit, the response of each standard is mathematically defined and then organized into standard classes that correspond to the error models used by the HP8510.

In conventional two-port calibration, three known impedance references and a single transmission standard are required. The most common coaxial standards are zeroelectrical-length short, shielded open, and matched load terminations, which all ideally have fixed magnitude and broadband phase response [4]. The accuracy of subsequent test device measurements is dependent on the following:

1) the accuracy of the test equipment,

2) how well the "known" devices (standards) are modeled, and

3) the exactness of the "error correction." 


\section{TRL (THRU-REFLECT-LINE)}

TRL refers to the three basic steps in the calibration process:

1) THRU - connection of port 1 and port 2, directly or with a short piece of transmission line.

2) REFLECT - connect identical one-port high reflection coefficient devices to each port.

3) LINE - insert a short length of transmission line between port 1 and port 2 (different line lengths are required for the THRU and LINE).

Unlike coaxial measurements, a set of three distinct impedance standards are often impossible to produce for noncoaxial transmission media. The new TRL calibration technique relies only on the characteristic impedance of a short transmission line. From two sets of two-port measurements that differ by this short length of transmission line and two reflection measurements, the full 12-term error model can be determined. Due to the simplicity of the calibration standards, TRL can be applied in dispersive transmission media such as microstrip, stripline, and waveguide. Vector error correction techniques can greatly reduce the effects of directivity, crosstalk, source match, and frequency response errors. The 12-term error model is used in both the "Full 2-Port" and the "TRL 2-Port" calibration types and provides the highest accuracy in common use today [5].

THRU-REFLECT-LINE is an approach to two-port calibration that relies on transmission lines rather than a set of discrete impedance standards. Although its mathematical derivation is different than the conventional "Full 2-Port," application of the technique results in the same 12-term error correctional model. While there is only one discrete standard required in the TRL calibration process compared to the conventional "Full 2Port" method, its mathematical solution is not quite as simple. A total of 16 measurements is required to quantify the 12 unknowns. Because there are more measurements than unknowns, two constants defining the calibration devices can also be determined. The complex reflection coefficient of the REFLECT standard and the propagation constant of the LINE are determined [6]. This is significant because these characteristics do not have to be specified. In other calibration approaches the resultant measurement accuracy is dependent on how well all of the standards are known. When applying TRL, accuracy is not compromised even though these characteristics are unknown. The characteristic impedance of the transmission LINE becomes the measurement reference and therefore has to be known or assumed ideal. 
There are three key advantages gained when using transmission lines as reference standards:

1) Transmission lines are among the simplest elements to realize in many noncoaxial media.

2) The impedance of transmission lines can be accurately determined from physical dimensions and materials.

3) Transmission lines have traditionally been used as standards and are well understood.

\section{TRL / WR340 Waveguide Window Measurement}

The process for implementing the TRL calibration method and measuring the 340 window will be described in five steps:

1) Selecting standards appropriate for the application that meet the basic requirements of the TRL technique.

2) Defining these standards for use with the HP8510 by modification of the internal calibration kit registers.

3) Performing the calibration.

4) Checking the performance.

5) Performing the WR340 waveguide window measurement.

\section{1 ) Selecting Appropriate Standards:}

A) THRU (zero length)

1) S21 and S12 are defined equal to 1 at zero degrees (typically used to set the reference plane).

B) REFLECT

1) Reflection coefficient magnitude need not be known.

2) Phase response need only be specified within a $1 / 4$ wavelength \pm 90 degrees either way.

3) Reflection standard must be the same on both ports.

C) LINE

1) Output impedance (Zout) of the LINE establishes the reference impedance after error correction is applied.

2) Attenuation of the LINE need not be known.

3) Optimal LINE length is $1 / 4$ wavelength or 90 degrees relative to the THRU at the center frequency.

4) Insertion phase of the LINE must never be the same as that of the THRU (zero or non-zero length). The insertion phase difference between the THRU and the LINE must be between 20 and 160 degrees. For example; if the THRU is zero degrees (not an offset 
THRU), then the LINE must measure between 20 and 160 degrees for the calibration to be considered usable. Measurement uncertainty will increase significantly when the insertion phase nears 0 or an integer multiple of 180 degrees [6].

\section{2 ) Defining Standards and Modifying Calibration Kit Registers:}

a) THRU - A zero length thru will be used.

b) REFLECT - Zero offset shorts, use WR340 waveguide blanking plates.

c) LINE - A existing 14.9-cm WR340 waveguide section will be used.

-WR340 waveguide upper and lower cut-off frequencies:

f cut-off (lower) $=\frac{c}{2 a}=\frac{2.997925 \times 10^{10} \mathrm{~cm} / \mathrm{s}}{2 \times 8.636 \mathrm{~cm}}=\mathbf{1 7 3 5 . 7} \mathbf{M H z}$

$\mathrm{a}=$ waveguide inside width (larger dia.) $=8.636 \mathrm{~cm}$ (3.4 inches)

f cut-off $($ upper $)=2 \times$ f cut-off $($ lower $)=3471.4 \mathbf{~ M H z}$

- Using a 14.9-cm waveguide section as the LINE:

Linear Delay $=\frac{l \sqrt{\varepsilon_{r}}}{c}=\frac{(0.149 \mathrm{~m}) \sqrt{1.000649}}{2.997925 \times 10^{8}} \times 10^{12}=497.2 \mathrm{ps}$

$l=$ offset length in meters

- In waveguide, group velocity varies with frequency due to dispersion as a function of the cut-off frequency.

$$
\begin{aligned}
& \text { Actual Delay }=\frac{\text { Linear Delay }}{\sqrt{1-(\mathrm{fco} / \mathrm{f})}}=\frac{497.2 \mathrm{ps}}{\sqrt{1-(1735.7 \mathrm{MHz} / 2856 \mathrm{MHz})^{2}}}=\mathbf{6 2 6 . 1} \mathbf{~ p s} \\
& \quad \mathrm{fco}=\text { lower cutoff frequency } \\
& \mathrm{f}=\text { measurement frequency }
\end{aligned}
$$


- TRL LINE insertion phase requirements are between 20 and 160 degrees.

Degrees (in the LINE) $=\frac{360 \times \mathrm{f}}{\mathrm{c}}\left(\sqrt{1-(\mathrm{fco} / \mathrm{f})^{2}}\right) l=$

$$
\frac{(360) 2.856 \times 10^{9} \mathrm{~Hz}}{2.997925 \times 10^{8} \mathrm{~m} / \mathrm{sec}}\left(\sqrt{\left.1-(1735.7 / 2856)^{2}\right)}(0.149 \mathrm{~m})=405.8\right. \text { degrees }
$$

405.8 degrees $=45.8$ degrees $(\operatorname{Mod} 360)$

- The following data must be entered into the internal calibration registers of the HP8510 thru the front panel or via computer to perform the "TRL Calibration:"

\begin{tabular}{|l|l|c|c|c|c|c|c|c|c|c|}
\hline \multicolumn{2}{|c|}{ Standard } & C0 & C1 & C2 & C3 & Offset & \multicolumn{2}{c|}{ Frequency } & $\begin{array}{c}\text { Coax or } \\
\text { WG }\end{array}$ & $\begin{array}{l}\text { Standard } \\
\text { Label }\end{array}$ \\
\hline No. & Type & L0 & L1 & L2 & L2 & $\begin{array}{c}\text { Delay } \\
\text { (ps) }\end{array}$ & Min. & Max. & & \\
\hline & & & & & & & & & & \\
\hline 14 & Delay & & & & & 0 & 1735.7 & 3471.4 & W & THRU \\
\hline 15 & Delay & & & & & 626.1 & 1735.7 & 3471.4 & W & LINE 1 \\
\hline 18 & Short & 0 & 0 & 0 & 0 & 0 & 1735.7 & 3471.4 & W & SHORT \\
\hline
\end{tabular}

\begin{tabular}{|l|l|l|l|}
\hline Standard Class Assignments & A & B & Standard Class Label \\
\hline & & & \\
\hline TRL THRU & 14 & & TRL THRU \\
\hline TRL REFLECT & 18 & & TRL SHORT \\
\hline TRL LINE & 15 & & LINE 14.9 \\
\hline
\end{tabular}

\section{3) Performing the Calibration:}

- The HP8510 network analyzer Port 1 and Port 2 are each connected to a " $7 \mathrm{~mm}$ to WR340 waveguide transition" via a 10-foot 7-mm "Gore - Phaseflex" phase stable cable. 
- Bolt a 7-inch WR340 waveguide straight section to each transition (not to be loosened or removed during this procedure). The straight sections will eliminate any evanescent wave effects caused by the transitions.

- The two waveguide straight pieces are then bolted together for a "thru measurement."

- The connection of these two waveguide straight pieces will serve as the "TRL Reference Plane.”

Procedure:

1) HP8510 - set center freq, freq span, number of points, and averaging if necessary. HP8510 press
a) $\mathrm{Cal}$
b) WR340 - TRL (newly installed TRL cal kit)
c) TRL 2-Port
d) Thru, TRL Thru

2) Disconnect junction of waveguide straight pieces and connect a WR340 blanking plate (short) to each waveguide piece (both ports).

HP8510 press
a) S11 Reflect, TRL Short
b) S22 Reflect, TRL Short
c) Isolation
d) Omit Isolation
e) Isolation Done

3) Disconnect both WR340 blanking plates and bolt the "14.9 cm LINE" waveguide section between both waveguide straight pieces.

HP8510 press
a) line/match, LINE 14.9
b) Save, TRL 2-Port
c) Select cal set

- The Gore cables, waveguide transitions, and waveguide straight sections will now be calibrated out of the upcoming measurements. 


\section{4) Checking the Calibration Performance:}

- Remove the "14.9 cm LINE" section and bolt the two waveguide straight pieces back together, which will allow the calibration to be checked.

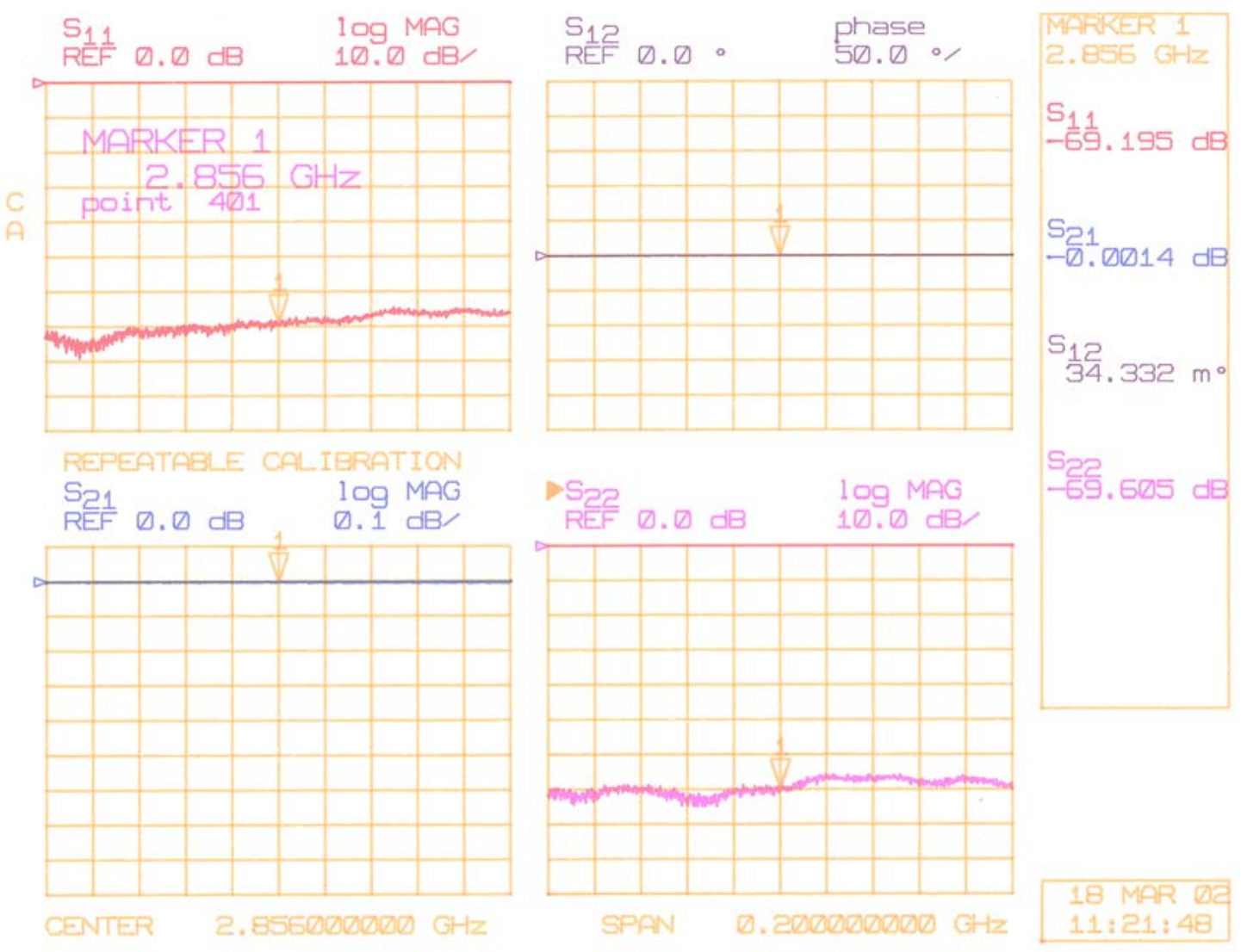

Fig. 1 - TRL Calibration

Figure 1 shows the calibration match in both directions ( $\mathrm{S} 11$ and S22) to be $-69 \mathrm{~dB}$ at $2856 \mathrm{MHz}$ with minimal insertion loss and the phase reference to be 0.03 degrees. 
- After the calibration is checked, the " $14.9 \mathrm{~cm}$ LINE" could be measured to compare against the calculated insertion phase. Reinstall the "14.9 cm LINE" between both waveguide straight sections.

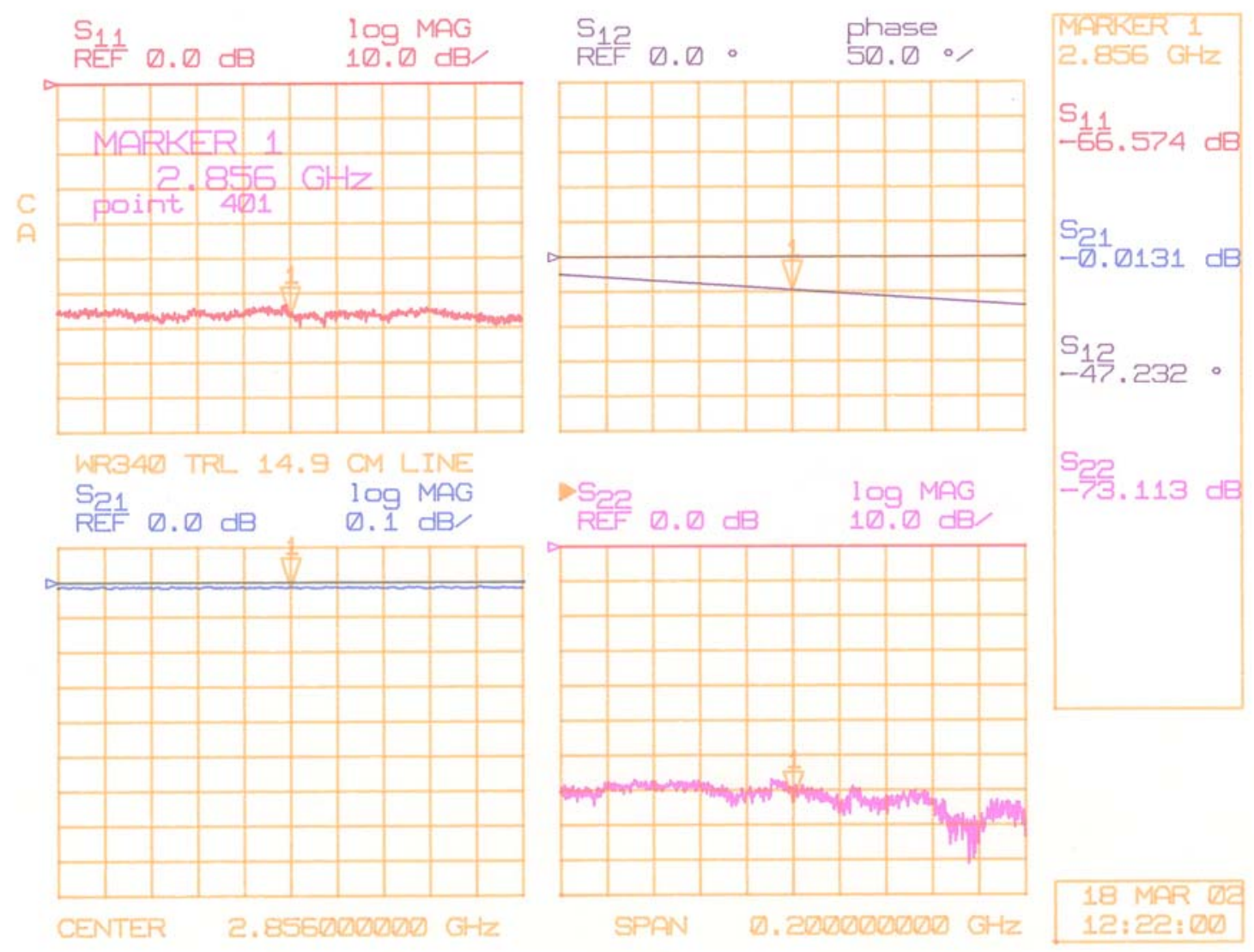

Fig. 2 - TRL $14.9 \mathrm{~cm}$ LINE

Figure 2 is a measurement of the " $14.9 \mathrm{~cm}$ LINE" waveguide section used as a device under test. The phase shift of the " $14.9 \mathrm{~cm}$ LINE" measures -47 degrees as compared to our previously calculated value of -46 degrees.

\section{5) Performing the WR340 Waveguide Window Measurement:}

- Remove the TRL "14.9cm LINE" and bolt the "WR340 Waveguide Window" between the two waveguide straight sections. 


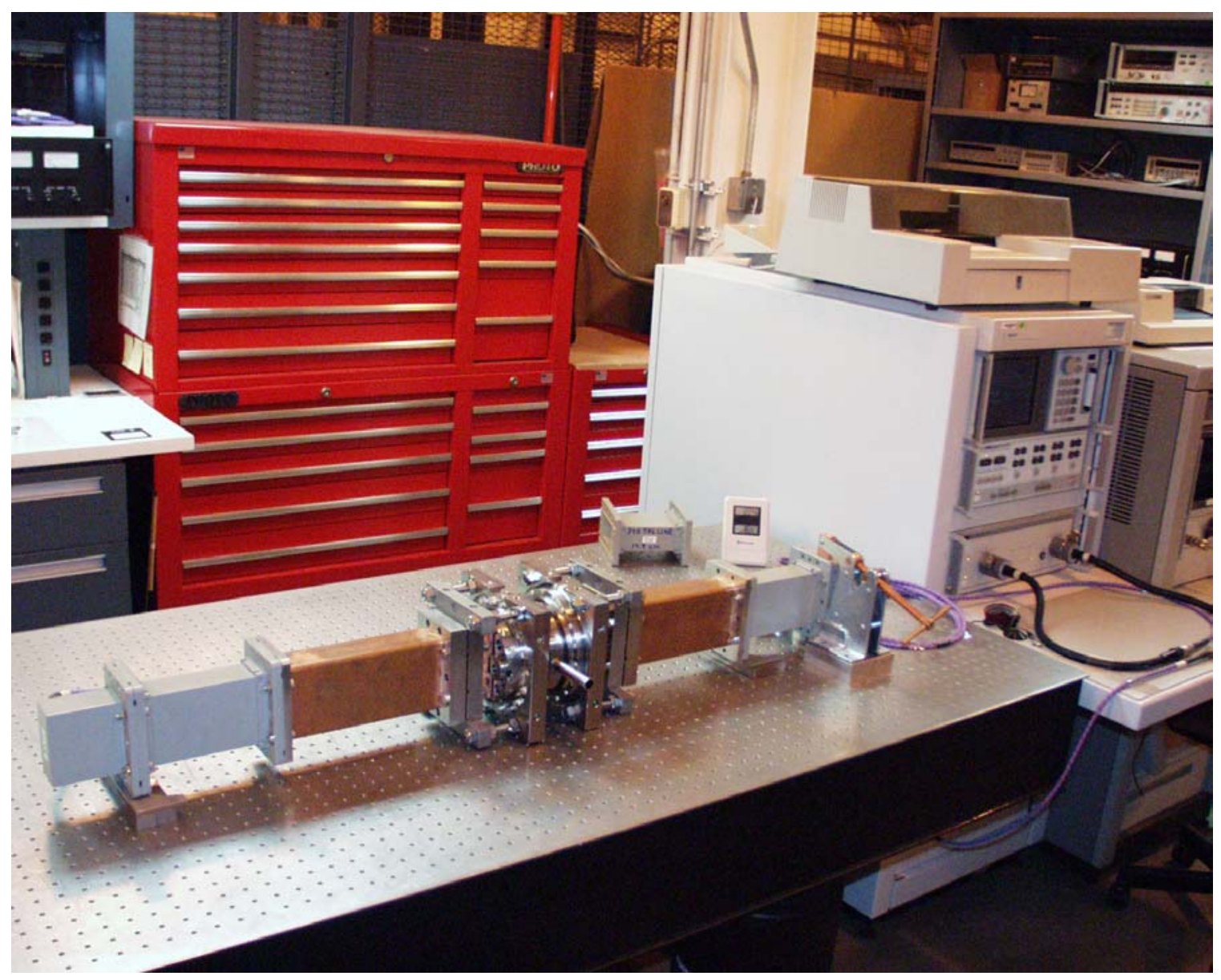

Fig. 3 - Window Measurement Setup

These additional techniques will be helpful in acquiring accurate data:

1) Calibration must be rechecked after the measurement is completed. A "repeatable calibration" (Fig. 1) is essential for assuring measurement accuracy.

2) Use minimal coaxial cable movement during the calibration and after the calibration until the measurement is completed. Figure 3 shows a Newport optic table with countersunk cut threads, allowing for "Port 1 test cable" and a "7mm to waveguide transition" to be shimmed to the right height and securely bolted before a calibration begins.

3) Two 10-foot "Gore Phaseflex" microwave cables are used to minimize effects caused by cable movements. These cables provide excellent phase and amplitude stability but do not suffer from the typical performance degradation associated with flexing traditionally designed flexible assemblies such as a standard Andrew heliax. 
4) Temperature and or humidity must be held constant from the time of the calibration until the measurement of the device under test is completed. If the measurement is not done under a controlled environment and temperature or humidity changes, a recalibration and new measurement may be necessary. If temperature or humidity changes and a "calibration recheck" performed after the measurement indicates the calibration to be the same as the original calibration, then new measurements are not necessary.

Note:

Errors caused by cable movement or temperature / humidity changes may be noticed in the calibration recheck. As an example of this error; consider Fig. 1 where the calibration recheck shows $\mathrm{S} 11$ or S22 calibrated return loss to be $-40 \mathrm{~dB}$ or $-50 \mathrm{~dB}$ instead of $\approx-69 \mathrm{~dB}$. This calibration would not be considered repeatable.
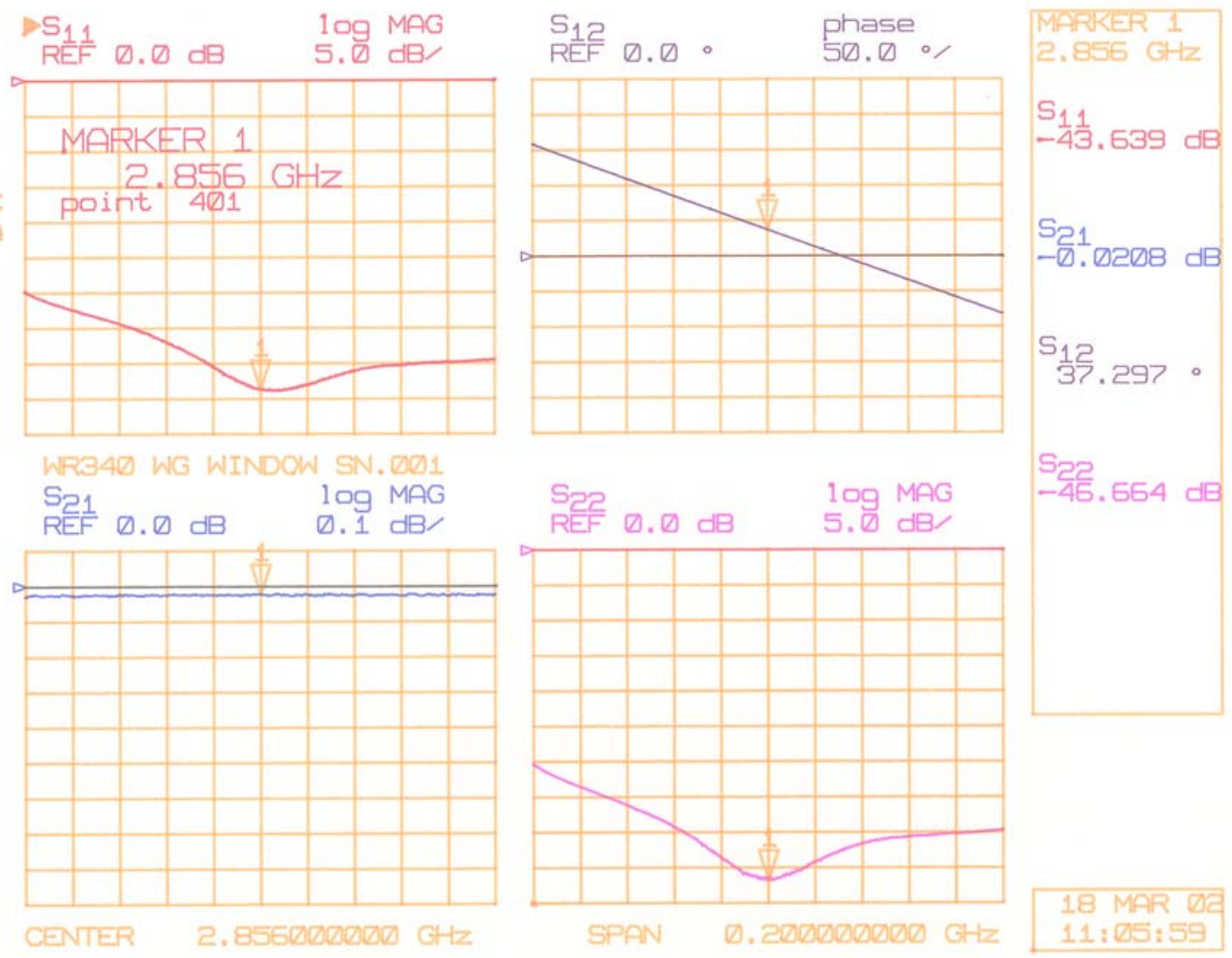

Fig. 4 - WR340 Waveguide Window (sn.001 )

Figure 4 shows the window return loss in both directions, $\mathrm{S} 11=-43.6 \mathrm{~dB}$ and $\mathrm{S} 22=$ $-46.6 \mathrm{~dB}$ at $2856 \mathrm{MHz}$ with an insertion loss of $0.02 \mathrm{~dB}$. 


\section{Conclusions}

With a typical "Full 2-Port Calibration" the open, load, and short calibration coefficients must be entered into the network analyzer registers. Over time and with usage if these coefficients no longer match the standards, an error will result. The "TRL 2-Port" calibration requires only a single impedance standard - a transmission line. Transmission lines have been traditionally used as standards and are among the simplest elements to realize in many noncoaxial media. The impedance of a transmission line can be accurately determined from physical dimensions and materials. An unknown high reflection device and a thru connection are sufficient to complete this TRL technique. In the TRL solution, the complex reflection coefficient of the REFLECT standard and the propagation constant of the LINE are determined. This is significant because now these characteristics do not have to be specified. In other calibration approaches the resultant measurement accuracy is dependent on how well all of the standards are known. When applying TRL, accuracy is not compromised even though these characteristics are unknown.

The TRL technique is not limited to dispersive transmission media and may also be used in making coaxial measurements. With precision coaxial transmission lines, TRL currently provides the highest accuracy in coaxial measurements available today.

\section{References}

[1] Agilent Technologies AN 1287-1, Understanding the Fundamental Principles of Vector Network Analysis, August, 2000.

[2] Agilent Technologies AN 1287-3, Applying Error Correction to Network Analyzer Measurements, July 25, 2001.

[3] Agilent Technologies 8510C Network Analyzer System Operating Manual, January, 1994.

[4] Hewlett Packard Product Note 8510-5A, Specifying Calibration Standards, February 1, 1988.

[5] Hewlett Packard Product Note 8510-13, Measuring Noninsertable Devices, August 1, 1988.

[6] Hewlett Packard Product Note 8510-8, Applying TRL Calibration, October 1, 1987. 